\title{
Interplay between Klebsiella pneumoniae producing KPC-31 and KPC-3 under treatment with high dosage meropenem: a case report
}

\author{
Gabriele Arcari $^{1}$ (D) Alessandra Oliva ${ }^{2}$ - Federica Sacco ${ }^{1,3}$ - Federica Maria Di Lella ${ }^{3} \cdot$ Giammarco Raponi $^{2,3}$ (1)

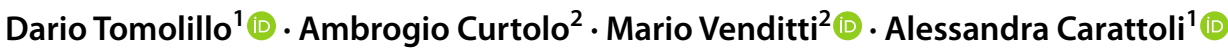

Received: 1 October 2021 / Accepted: 29 November 2021 / Published online: 6 January 2022

(c) The Author(s), under exclusive licence to Springer-Verlag GmbH Germany, part of Springer Nature 2021

\begin{abstract}
The objective was to study ceftazidime-avibactam resistant and susceptible Klebsiella pneumoniae isolated from a patient admitted to the Policlinico Umberto I of Rome for SARS-CoV2. Data on the evolution of patient's conditions, antimicrobial therapies, and microbiological data were collected. Whole-genome sequencing performed by Illumina and Nanopore sequencing methods were used to type the strains. During the hospitalization, a SARS-CoV2-infected patient was colonized by a KPC-producing $K$. pneumoniae strain and empirically treated with ceftazidime-avibactam (CZA) when presenting spiking fever symptoms. Successively, ST2502 CZA-resistant strain producing the KPC-31 variant gave a pulmonary infection to the patient. The infection was treated with high doses of meropenem. The KPC-31-producing strain disappeared but the patient remained colonized by a KPC-3-producing K. pneumoniae strain. An interplay between highly conserved KPC-31and KPC-3-producing ST2502 strains occurred in the SARS-CoV2 patient during the hospitalization, selected by CZA and carbapenem treatments, respectively.
\end{abstract}

Keywords Klebsiella pneumoniae $\cdot$ Ceftazidime-avibactam $\cdot$ KPC

Multidrug-resistant Enterobacterales represent a therapeutic challenge due to their global spread and limited therapeutic options. Klebsiella pneumoniae carbapenemase (KPC) is the most prevalent enzyme in the Americas, the Mediterranean countries, and China, conferring resistance against several antimicrobial agents commonly used against gram-negative bacteria [1]. In 2018, a new antimicrobial combination, ceftazidime/avibactam (CZA), was approved against carbapenemase-producing Enterobacterales and become rapidly the first-line therapeutic option in carbapenem-producing Enterobacterales producing class $\mathrm{A}$, class $\mathrm{C}$, and some class $\mathrm{D}$ beta-lactamase. While CZA was being used more and more, the first resistances were reported [2]. Interestingly, it was found that during CZA administration, the MIC of

Alessandra Carattoli

alessandra.carattoli@uniroma1.it

1 Department of Molecular Medicine, Sapienza University of Rome, Rome, Italy

2 Department of Public Health and Infectious Diseases, Sapienza University of Rome, Rome, Italy

3 Microbiology and Virology Unit, University Hospital Policlinico Umberto I, Rome, Italy meropenem (MEM) could decrease and some KPC-producing Klebsiella pneumoniae (KPC-Kp) strains could result CZA-resistant and MEM-susceptible [3, 4].

In November 2020, a 59-year-old man was admitted to the Emergency Department of Policlinico Umberto I of Rome for previously diagnosed severe acute respiratory syndrome coronavirus 2 (SARS-CoV2) infection and shortness of breath (day 0, Fig. 1). Medical history was notable for type-2 diabetes, arterial hypertension, obesity (BMI 32), and persistent atrial fibrillation. Rapidly worsening dyspnea and analysis of arterial blood gas demonstrated acute respiratory failure with severe hypoxemia requiring high flow oxygen delivery with helmet continuous positive airway pressure. Initial prescription included dexamethasone, enoxaparin, ceftriaxone, and azithromycin, while remdesivir was not prescribable due to symptoms onset lasting over ten days, as for local guidelines. Patient's conditions rapidly deteriorated necessitating endotracheal intubation before being transferred to the intensive care unit (ICU). On day 4 of hospitalization, empiric therapy with linezolid $600 \mathrm{mg}$ bid and piperacillin/tazobactam $4.5 \mathrm{~g}$ qid was started due to spiking fever. This treatment was early interrupted because 


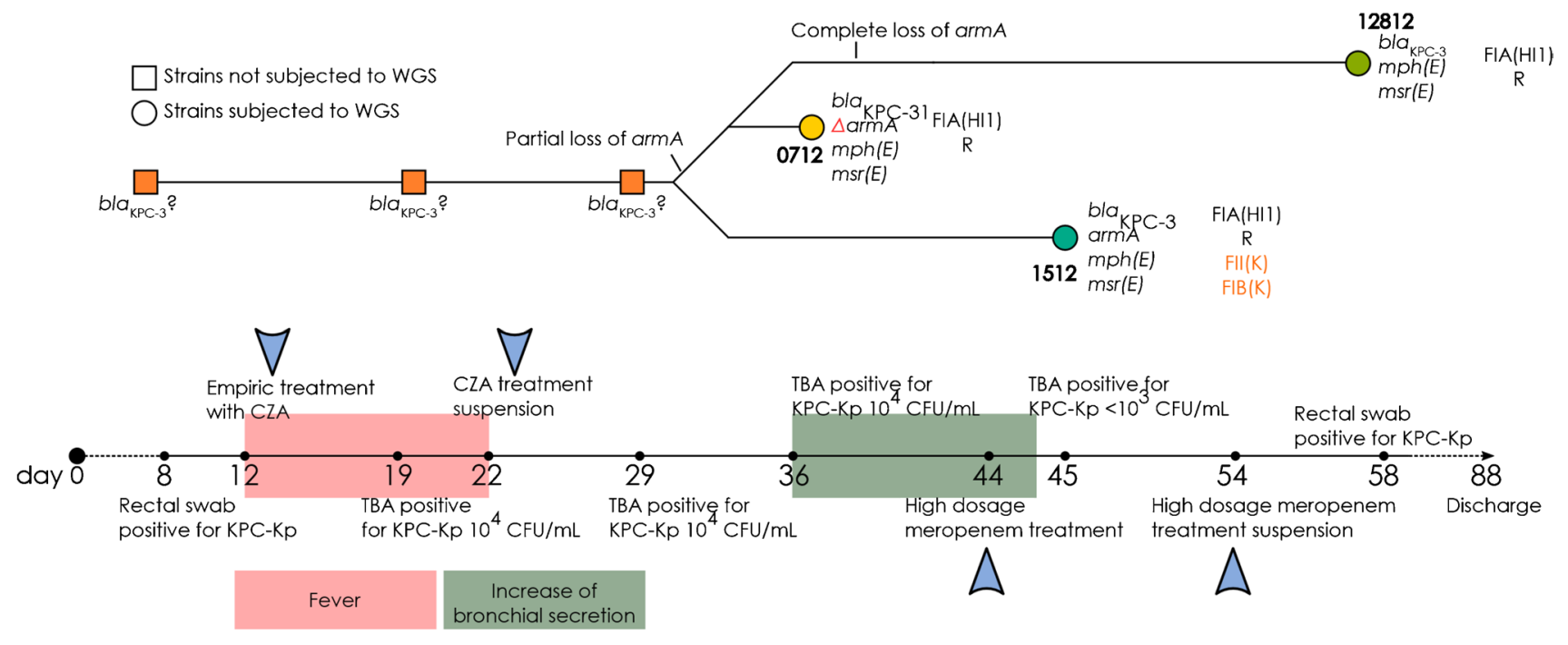

Fig. 1 Timeline of the KPC-producing strains involved in the case report. The upper part of the figure represents the isolated strains, their evolution, and the main characteristics of those subjected to whole-genome sequencing (i.e., resistome and replicon content of the

of no growth on blood cultures (BCs), negative values of procalcitonin (PCT), and resolution of fever.

On day 8, the routine rectal swab for surveillance of carbapenem-resistant Enterobacterales performed by directly plating a rectal swab on a carbaenterochrom agar plate (Microbiol s.r.l., Macchiareddu, Italy) tested positive for KPC-Kp. This strain was reported as susceptible to colistin $(\mathrm{MIC} \leq 0.5 \mu \mathrm{g} / \mathrm{ml}$ ), trimethoprim/sulfamethoxazole (MIC $\leq 20 \mu \mathrm{g} / \mathrm{ml}$ ), and CZA (MIC $4 \mu \mathrm{g} / \mathrm{ml}$ ) by the Vitek2 automated test (bioMérieux, Marcy l'Etoile, France; Suppl. Table 1). On day 11, he was extubated and started on noninvasive positive pressure ventilation.

On day 12, due to the new onset of spiking fever, empiric treatment with CZA $2.5 \mathrm{~g}$ tid was started after drawing BCs. Subsequently, considering the persistence of fever, it was decided to remove and substitute the central venous catheter, with tip culture showing growth of oxacillin-resistant Staphylococcus aureus and Candida albicans. Treatment was then implemented with daptomycin and caspofungin (from day 20), obtaining resolution of fever and progressive improvement of clinical conditions. CZA was interrupted on day 22 after 10 days of administration. Follow-up BCs and echocardiography tested negative; daptomycin and caspofungin were administered for a total of 14 days.

From day 36, a progressive increase of bronchial secretions was noted with slight worsening of respiratory function and difficult weaning from the non-invasive ventilation. The surveillance culture of the tracheal aspirate reported the growth of $>1 \times 10^{5} \mathrm{CFU} / \mathrm{ml}$ of KPC-Kp, showing imipenem (IMI) $\mathrm{MIC} \leq 0.25 \mu \mathrm{g} / \mathrm{ml}$, and meropenem (MEM) MIC $\leq 1 \mu \mathrm{g} / \mathrm{ml}$ (strain 0712). MIC values for CZA, bla $a_{\mathrm{KPC}}$ carrying plasmids). In the lower part, black dots with dates represent noteworthy events in the case, blue arrows represent the start and the end of pertinent therapies, and colored blocks over the line represent major clinical events

meropenem/vaborbactam (M/V), and imipenem/relebac$\operatorname{tam}(\mathrm{I} / \mathrm{R})$ were evaluated by concentration gradient method (MIC Test Strip, Liofilchem srl, Italy) and by broth microdilution method (SensititreTM EUMDROXF, Thermo Fisher Scientific, Waltham, MA), on Mueller-Hinton Agar plates (bioMérieux, Marcy l'Etoile, France). The broth microdilution panel contained M/V $(0.06 / 8-16 / 8 \mu \mathrm{g} / \mathrm{mL}), \mathrm{I} / \mathrm{R}$ $(0.06 / 4-8 / 4 \mu \mathrm{g} / \mathrm{mL})$, and CZA $(0.25 / 4-16 / 4 \mu \mathrm{g} / \mathrm{mL})$. The 0712 strain resulted resistant to CZA (MIC $\geq 256 / 4 \mu \mathrm{g} / \mathrm{ml}$ ) but susceptible to both $\mathrm{M} / \mathrm{V}(\mathrm{MIC}=0.38 / 8 \mu \mathrm{g} / \mathrm{ml})$ and $\mathrm{I} / \mathrm{R}$ $(\mathrm{MIC}=0.50 / 4 \mu \mathrm{g} / \mathrm{ml}$; Suppl. Table 1).

On day 41, a chest X-ray showed the appearance of new and confluent mid-basal consolidations and meropenem $1 \mathrm{~g}$ bolus followed by high dose; 3-h extended infusion of $2 \mathrm{~g}$ tid was started, prompt early ( $48 \mathrm{~h}$ ) clinical improvement.

Under MEM treatment, on day 45, a new culture of tracheal aspirate showed absence of the previously isolated CZA-resistant KPC-Kp but reported the growth of few colonies $\left(<1 \times 10^{3} \mathrm{CFU} / \mathrm{ml}\right)$ of a MEM-resistant CZA-susceptible KPC-Kp (IMI MIC $\geq 16 \mu \mathrm{g} / \mathrm{ml}$; MEM MIC $\geq 16 \mu \mathrm{g} /$ $\mathrm{ml}, \mathrm{CZA} \mathrm{MIC}=4 \mu \mathrm{g} / \mathrm{ml}, \mathrm{M} / \mathrm{V} \mathrm{MIC}=0.38 / 8 \mu \mathrm{g} / \mathrm{ml}, \mathrm{I} / \mathrm{R}$ $\mathrm{MIC}=0.50 / 4 \mu \mathrm{g} / \mathrm{ml}$; strain 1512).

Treatment with MEM was carried on for a total of 10 days, with no adverse effects. Respiratory function improved significantly, allowing progressive reduction of oxygen delivery until suspension on day 65 and transfer to a general ward.

On day 58, the culture of rectal swab showed colonization by a carbapenem-resistant KPC-Kp (IMI MIC $\geq 16 \mu \mathrm{g} /$ $\mathrm{ml}$; MEM MIC $\geq 16 \mu \mathrm{g} / \mathrm{ml}, \mathrm{CZA}$ MIC $=4 \mu \mathrm{g} / \mathrm{ml}, \mathrm{M} / \mathrm{V}$ $\mathrm{MIC}=0.5 / 8 \mu \mathrm{g} / \mathrm{ml}, \mathrm{I} / \mathrm{R} \mathrm{MIC}=0.75 / 4 \mu \mathrm{g} / \mathrm{ml} ;$ strain 12812). 
On day 88 , the patient was discharged to a rehabilitation center.

Whole-genome sequencing (WGS) was obtained as previously described [3] by the Illumina MiSeq instrument (Illumina Inc., San Diego, CA, USA) for three K. pneumoniae strains, namely the first strain isolated from tracheal aspirate after the empiric treatment with CZA (0712), the strain isolated from tracheal aspirate in the fourth day after the beginning of the high-dosage (3-h extended infusion of $2 \mathrm{~g}$ tid) MEM treatment (1512), and the strain colonizing the patient 17 days after the beginning of the high-dosage MEM treatment (12812). The three strains were also subjected to Nanopore sequencing into the MinION Flow Cell (R9.4.1) following SQK-RBK004 sequencing procedures in an Mk1C MinION platform [5].

In silico WGS studies performed using Kleborate [6] demonstrated that the 3 isolates were ST2502. This peculiar ST belongs to the same clonal complex of the ST101 and is considered to be hyperendemic in certain areas of the Tuscany region [7].

A phylogenetic analysis of ST2502 and ST101 clones was performed comparing genomes sequenced in this study with those downloaded from GenBank and from the Institut Pasteur (https://bigsdb.pasteur.fr/) databases or sequenced in previous studies $[5,8]$. The genomes were annotated using Prokka [9] and the resulting general feature formats (GFFs) were analyzed using Roary v3.11.3 [10] to identify the core and the accessory genes and to obtain a core genome alignment. Recombining regions removal was carried out by the Gubbins algorithm [11] (https://usegalaxy.eu/) generating a maximum likelihood phylogenetic tree with RAxML using default parameters [12].

The three strains were each other highly related at chromosomal level (Fig. 2; 0-1 SNPs in the core genome) but differed for the resistance gene content. Complete circular plasmid assemblies were obtained for strains $0712(69,857 \mathrm{bp}$; GenBank Acc. No. MZ606382) and 12812 (69,171 bp, Acc, No. MZ606380) by using the Unicycler tool with the bold bridging mode (https://usegalaxy.eu/ server). The complete circular plasmid sequence of 264,856 bp from strain 1512 was obtained by the Flye assembly on nanopore long-reads but was not reconfirmed by Unicycler.

Plasmid analysis demonstrated that all strains carried the same plasmid characterized by the presence of FIA(HI1) and $\mathrm{R}$ replicons (Fig. 3, visualized using the Circos [13] tool).

In strain 0712, the FIA(HI1)-R plasmid encoded the CZA-resistant KPC-31 variant. The $b l a_{\mathrm{KPC}-31}$ gene was identified in the Tn4401 transposon; a deleted $\triangle a r m A$ gene

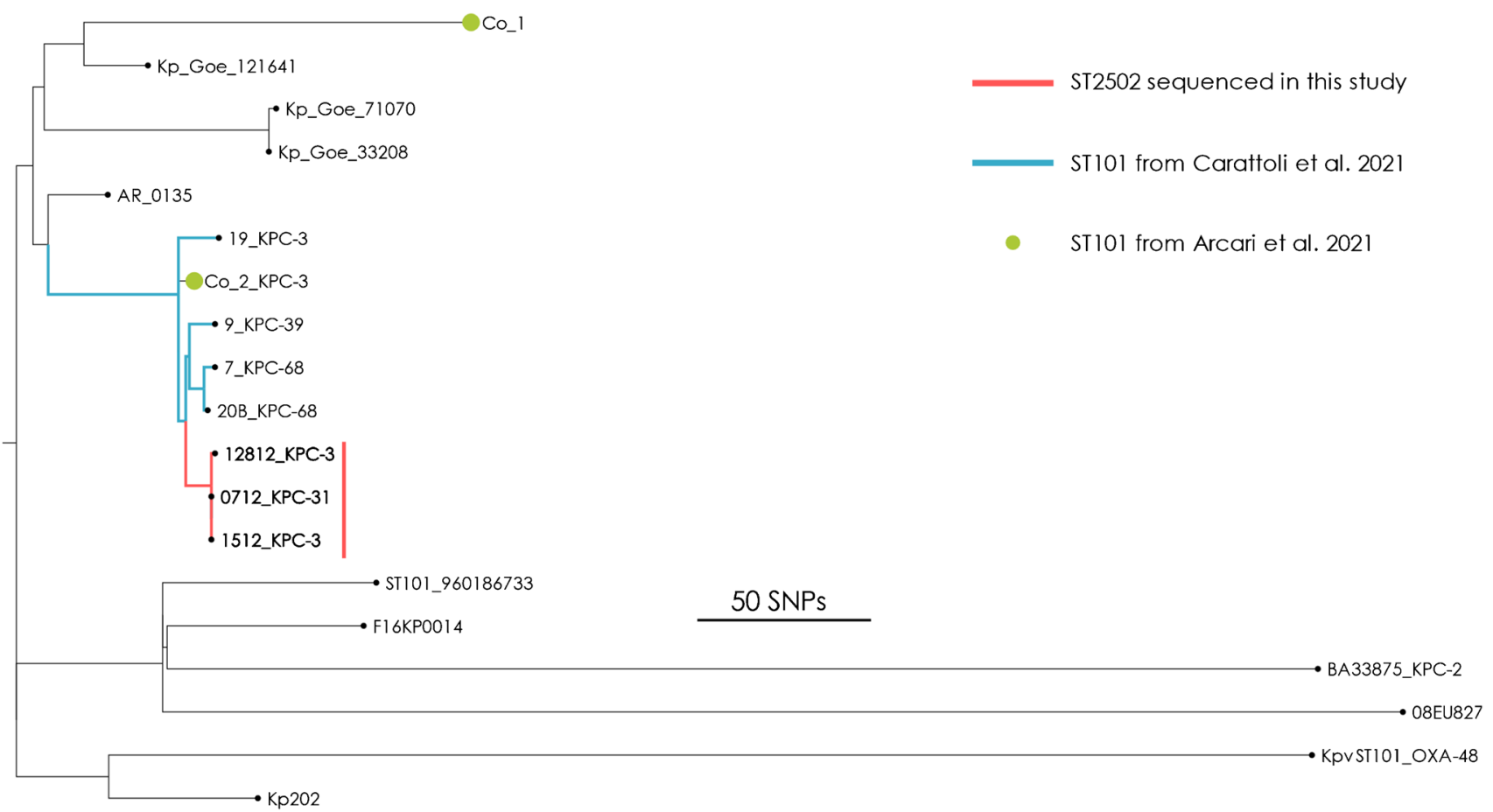

Fig. 2 Phylogenetic tree based on a core genome alignment of 0712, 1512, and 12812 ST2502 strains in comparison with ST101 Klebsiella pneumoniae strains. The figure shows an unrooted maximum likelihood phylogenetic tree based on a concatenated core gene alignment of $19 \mathrm{~K}$. pneumoniae genomes belonging to the ST101 and to the highly related ST2502. Highlighted by the orange circle, the three strains sequenced in this study, the other genomes were downloaded from the GenBank and from the Institut Pasteur databases or were sequenced in previous studies (Strains Co_1 and Co_2 from Arcari et al. 2021 [7], and strains 19, 9, 7, and 20B from Carattoli et al. 2021 [3]). The visualization of the tree was performed with FigTree 
Fig. 3 Circos plot of the $b l a_{\mathrm{KPC}}$ carrying plasmids. Schematic representation of the three plasmids involved in the study. Starting from the outside to the inside of the plot, the main features of the plasmids (i.e., replicon content, resistance genes, and, for the 1512 plasmid, the genes involved in the fusion between the FIA(HI1)-R and the pKPN plasmids), the GCskew, and a synteny between the plasmids. Specifically, in green the alignments between the 0712 and 12812 plasmids while in blue the alignments with the 1512 strain

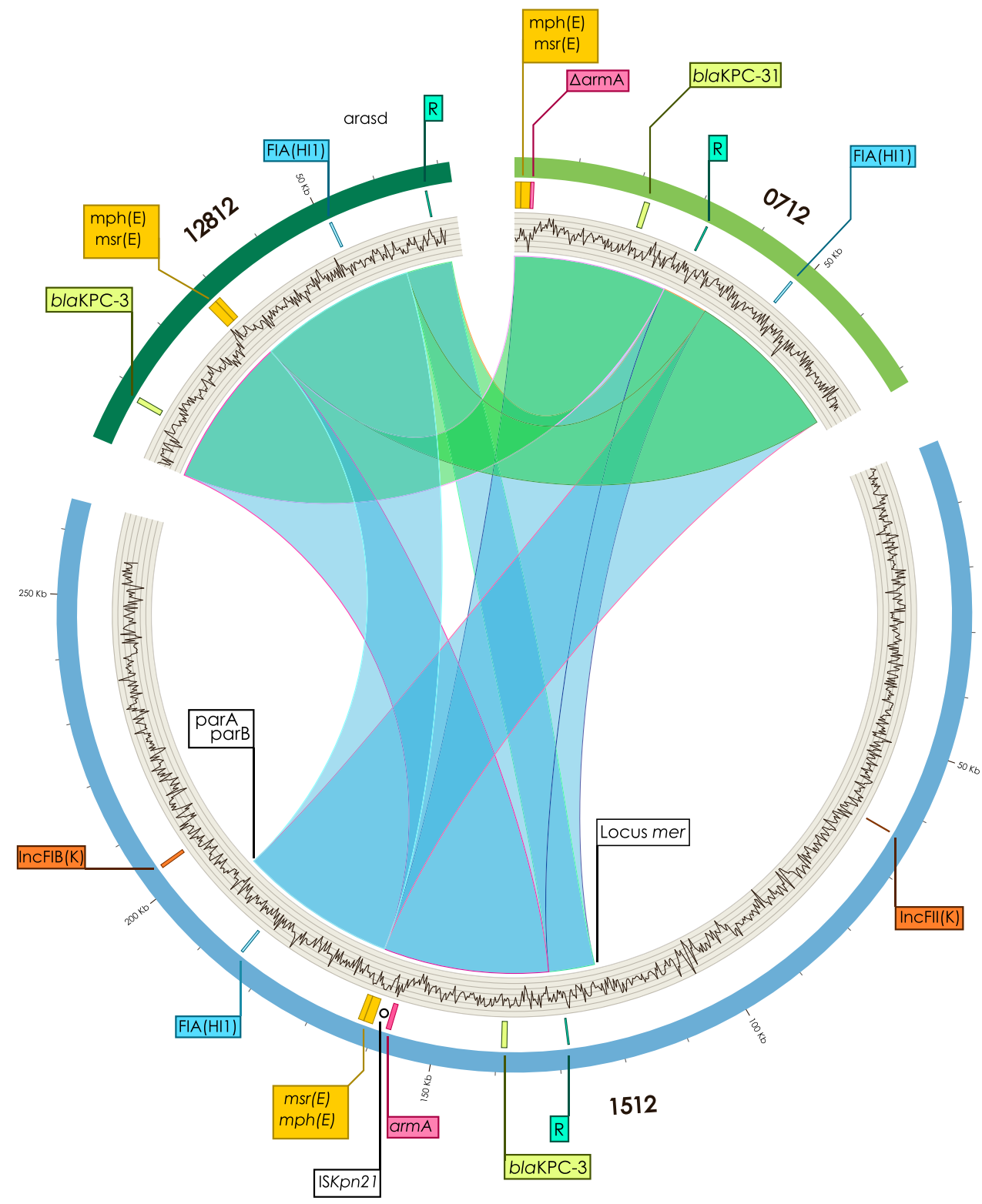

and the macrolide resistance $m p h E$ - $m s r E$ genes were also present of the plasmid.

In strain 1512, the FIA(HI)-R plasmid carried the $b l a_{\mathrm{KPC}-3}$ carbapenem resistance gene in the Tn4401 transposon, an intact copy of the armA gene encoding the $16 \mathrm{~S}$ rRNA methylase, conferring resistance to all aminoglycosides and the macrolide resistance $m p h E-m s r E$ genes. The FIA(HI1)-R plasmid was fused with the pKPN plasmid identified by the FIIk-FIBk replicons.

Finally, in strain 12812, the FIA(HI1)-R plasmid carried the $b l a_{\mathrm{KPC}-3}$ gene and $m p h E-m s r E$ macrolide resistance genes but has completely lost the armA gene. The pKPN plasmid was also identified in this strain but not in fusion with FIA(HI1)-R.
In conclusion, the patient was initially colonized by a KPC-3-producing $K$. pneumoniae strain. Empiric CZA therapy was started at the onset of a spiking fever based on the observation that the patient was colonized by a CZA-susceptible strain and interrupted after 10 days of administration. This treatment presumably selected for a ST2502 producing the KPC-31 variant, which silently colonized the patient. KPC-31, showing the D179Y amino acid substitution located in the $\Omega$ loop of the protein, has been the most frequently reported CZA-resistant KPC-3 variant worldwide [14-18]. This variant confers a CZAresistant phenotype associated with susceptibility or decreased resistance to carbapenems. The ST2502 producing KPC-31 (strain 0712) gave a pulmonary infection 
to the patient 14 days after the suspension of the empiric CZA treatment. The infection was treated with high doses of MEM since the isolate was carbapenem susceptible. The decision of using high dose of MEM was based on the need of achieving adequate pharmacokinetic and pharmacodynamic target attainment and possibly reduce the chances of development of resistance [19].

The KPC-31-producing strain disappeared after 4 days of MEM treatment, along with a rapid amelioration of patient's clinical conditions, and was not cultivated from other samples during the entire hospitalization time. However, the culture of tracheal aspirate reported the growth, at very low concentration $\left(<1 \times 10^{3} \mathrm{CFU} / \mathrm{ml}\right)$, of ST2502 producing the carbapenemase KPC-3 (strain 1512). The high-dose MEM therapy cured the infection, but the $K$. pneumoniae was not completely cleared from the patient. In fact, the ST2502 producing KPC-3 still colonized the intestinal tract of the patient, many days after the end of the MEM therapy (strain 12812).

The interplay between the $b l a_{\mathrm{KPC}-3}$ and $b l a_{\mathrm{KPC}-31}$ genes occurred within the same ST2502 clone on highly related plasmids of the FIA(HI1)-R type, previously described in ST101 strains from the same hospital [3]. These plasmids contributed to the evolution of the clone by hosting the $b l a_{\mathrm{KPC}}$ variants but also underwent to rearrangement (gain or loss of additional resistance determinants carrying the armA gene) and fusion with co-resident plasmids. This study confirms how difficult is the treatment of infections sustained by KPC-Kp that can rapidly evolve from CZA-susceptible to CZA-resistant under CZA-treatment by KPC-3 to KPC-31 mutation. KPC-31-producing strains can be treated with MEM, but still in the patient can persist colonization of MEM-resistant KPC-3 producers.

Supplementary Information The online version contains supplementary material available at https://doi.org/10.1007/s10096-021-04388-y.

Author contribution G. Arcari, A. Carattoli, A. Oliva, M. Venditti: conceived and designed the analysis, contributed data, wrote the paper; F. Sacco, F. M. Di Lella, D. Tomolillo, and A. Curtolo collected the data and performed the analysis.

Funding This work was supported by Sapienza University of Rome, Top Scientist 1\% Funds 2019 to Alessandra Carattoli, and Ateneo Funds 2019 no. RM11916B6A8B5F8D to Giammarco Raponi.

Data availability Complete circular plasmid assembly for strains 0712 and 12812 were released under GenBank Acc. No. MZ606382 and Acc. No. MZ606380, respectively. SRA and Biosamples of strains 0712, 12,812, and 1512 were released at NCBI Bioproject PRJNA746285; whole genome sequences were released under Bioproject PRJNA746288.

\section{Declarations}

Ethics approval This research was conducted according to the guidelines of the Declaration of Helsinki, and approved by the Institutional Review Board (or Ethics Committee) of Policlinico Umberto I of Rome. The informed consent form has been signed by the patient.

Conflict of interest The authors declare no competing interests.

\section{References}

1. Bush K, Bradford PA (2020) Epidemiology of $\beta$-lactamaseproducing pathogens. Clin Microbiol Rev 33:1-37. https://doi. org/10.1128/CMR.00047-19

2. Di Bella S, Giacobbe DR, Maraolo AE, Viaggi V, Luzzati R, Bassetti M, et al (2021) Resistance to ceftazidime/avibactam in infections and colonisations by KPC-producing Enterobacterales: a systematic review of observational clinical studies. J. Glob. Antimicrob. Resist. [Internet] 25:268-81. doi: https://doi.org/10. 1016/j.jgar.2021.04.001

3. Shields RK, Nguyen MH, Press EG, Chen L, Kreiswirth BN, Clancy CJ (2017) Emergence of ceftazidime-avibactam resistance and restoration of carbapenem susceptibility in Klebsiella pneumoniae carbapenemase- producing $\mathrm{K}$ pneumoniae: a case report and review of literature. Open Forum Infect Dis 4(3):1-4. https://doi.org/10.1093/ofid/ofx101

4. Haidar G, Clancy CJ, Shields RK, Hao B, Cheng S, Nguyen MH (2017) Mutations in blaKPC-3 that confer ceftazidime-avibactam resistance encode novel KPC-3 variants that function as extendedspectrum $\beta$-lactamases. Antimicrob Agents Chemother. https:// doi.org/10.1128/AAC.02534-16

5. Carattoli A, Arcari G, Bibbolino G, Sacco F, Tomolillo D, Di Lella FM, et al (2021) Evolutionary trajectories toward ceftazidime-avibactam resistance in Klebsiella pneumoniae clinical isolates. Antimicrob. Agents Chemother 65 https://doi.org/10.1128/ aac.00574-21

6. Lam MMC, Wick RR, Watts SC, Cerdeira LT, Wyres KL, Holt KE (2021) A genomic surveillance framework and genotyping tool for Klebsiella pneumoniae and its related species complex. Nat. Commun. [Internet] 12 https://doi.org/10.1038/s41467-021-24448-3

7. Arena F, Di Pilato V, Vannetti F, Fabbri L, Antonelli A, Coppi M, et al (2020) Population structure of kpc carbapenemase-producing klebsiella pneumoniae in a long-term acute-care rehabilitation facility: identification of a new lineage of clonal group 101, associated with local hyperendemicity. Microb. Genomics 6 https:// doi.org/10.1099/mgen.0.000308

8. Arcari G, Raponi G, Sacco F, Bibbolino G, Di Lella FM, Alessandri F, et al (2020) Klebsiella pneumoniae infections in COVID-19 patients: a 2-month retrospective analysis in an Italian hospital. Int. J. Antimicrob. Agents

9. Seemann T (2014) Prokka: rapid prokaryotic genome annotation. Bioinformatics 30:2068-2069. https://doi.org/10.1093/bioinforma tics/btu 153

10. Page AJ, Cummins CA, Hunt M, Wong VK, Reuter S, Holden MTG et al (2015) Roary: rapid large-scale prokaryote pan genome analysis. Bioinformatics 31:3691-3693. https://doi.org/10.1093/ bioinformatics/btv421

11. Croucher NJ, Page AJ, Connor TR, Delaney AJ, Keane JA, Bentley SD et al (2015) Rapid phylogenetic analysis of large samples of recombinant bacterial whole genome sequences using Gubbins. Nucleic Acids Res 43:e15. https://doi.org/10.1093/nar/gku1196 
12. Stamatakis A (2014) RAxML version 8: a tool for phylogenetic analysis and post-analysis of large phylogenies. Bioinformatics 30:1312-1313. https://doi.org/10.1093/bioinformatics/btu033

13. Krzywinski M, Schein J, Birol I, Connors J, Gascoyne R, Horsman $\mathrm{D}$ et al (2009) Circos: an information aesthetic for comparative genomics. Genome Res 19:1639-1645. https://doi.org/10.1101/ gr.092759.109

14. Gaibani P, Re MC, Campoli C, Viale PL, Ambretti S (2020) Bloodstream infection caused by KPC-producing Klebsiella pneumoniae resistant to ceftazidime/avibactam: epidemiology and genomic characterization. Clin Microbiol Infect [Internet] 26:516.e1-516.e4. https://doi.org/10.1016/j.cmi.2019.11.011

15. Livermore DM, Warner M, Jamrozy D, Mushtaq S, Nichols WW, Mustafa $\mathrm{N}$ et al (2015) In vitro selection of ceftazidime-avibactam resistance in enterobacteriaceae with KPC-3 carbapenemase. Antimicrob Agents Chemother 59:5324-5330. https://doi.org/10. 1128/AAC.00678-15

16. Shields RK, Chen L, Cheng S, Chavda KD, Press EG, Snyder A, et al (2017) Emergence of ceftazidime-avibactam resistance due to plasmid-borne blaKPC-3 mutations during treatment of carbapenem-resistant Klebsiella pneumoniae infections. Antimicrob. Agents Chemother 61.
17. Zhang P, Shi Q, Hu H, Hong B, Wu X, Du X et al (2020) Emergence of ceftazidime/avibactam resistance in carbapenem-resistant Klebsiella pneumoniae in China. Clin Microbiol Infect 26:124. e1-124.e4. https://doi.org/10.1016/j.cmi.2019.08.020

18. Shapiro AB, Moussa SH, Carter NM, Gao N, Miller AA (2021) Ceftazidime-avibactam resistance mutations V240G, D179Y, and D179Y/T243M in KPC-3 $\beta$-lactamase do not alter cefpodoximeETX1317 susceptibility. ACS Infect Dis 7:79-87. https://doi.org/ 10.1021/acsinfecdis.0c00575

19. Tam VH, Chang KT, Zhou J, Ledesma KR, Phe K, Gao S et al (2017) Determining $\beta$-lactam exposure threshold to suppress resistance development in Gram-negative bacteria. J Antimicrob Chemother 72:1421-1428. https://doi.org/10.1093/jac/dkx001

Publisher's Note Springer Nature remains neutral with regard to jurisdictional claims in published maps and institutional affiliations. 